\title{
Parent Perceived Impact of Spaniard Boys' and Girls' Inattention, Hyperactivity, and Oppositional Defiant Behaviors on Family Life
}

Journal of Attention Disorders

14(3) 247-255

(c) 2010 SAGE Publications

Reprints and permission:

sagepub.com/journalsPermissions.nav

DOI: $10.1 / 77 / 1087054709347 / 80$

http://jad.sagepub.com

(SAGE

\author{
José J. Bauermeister, ' Aníbal Puente, ${ }^{2}$ José V. Martínez, ${ }^{3}$ Eduardo Cumba,' \\ Rubén O.Scándar, ${ }^{4}$ and José A. Bauermeister ${ }^{5}$
}

\begin{abstract}
Objective: This study examined the impact of inattention, hyperactivity, and oppositional defiant disorder (ODD) behaviors and gender on family life. Method: We created scales for the Family Experiences Inventory (FEI) in a nonclinical sample of Spaniard families with children ages 6 to 12 years $(N=369)$ and analyzed the perceived impact of these three behavior dimensions on family experiences. Results: Multiple regression analyses indicated that ODD behaviors were uniquely correlated with Total FEl and its dimensions. Inattention was also uniquely related to higher negative Impact on School Relations and lower Positive Impact on Parents scales. Finally, gender-hyperactivity interactions indicated that boys with higher hyperactivity scores were more likely to score higher on the FEl Total, School Relations, and Siblings scales, and more likely to score lower on the Positive Impact on Parents scale than girls. Conclusions: These findings suggested that parents perceive greater child-related impact and place greater burden from having a male child with hyperactivity. Inattention, hyperactivity, and oppositional defiant behaviors are associated with global parent-child interactive stress but the pattern of associations will vary depending upon the behavior, child gender, and context of family life examined.
\end{abstract}

\section{Keywords}

attention-deficit/hyperactivity disorder; ODD; stress; gender; Latino/Hispanics

The association between parental stress and ADHD and oppositional defiant disorder (ODD) in children has been identified in the literature (Anastopoulos, Guevremont, Shelton, \& DuPaul, 1992; Breen \& Barkley, 1988; Harrison \& Sofronoff, 2002; Podolski \& Nigg, 2001). ADHD is a developmental disorder characterized by a persistent pattern of inattention and/or hyperactivity-impulsivity. ODD, on the other hand, consists of a recurrent pattern of negativistic, defiant, disobedient, and hostile behaviors toward authority figures (American Psychiatric Association, 1994). Although researchers have suggested that inattention, hyperactivity, and oppositional defiance are relatively independent dimensions (Bauermeister, 1992; Hinshaw, 1987; Pelham, Gnagy, Greenslade, \& Milich, 1992; Wolraich et al., 2003) with partially different etiologies and correlates (Hinshaw, 1987; Hinshaw \& Lee, 2003), it is not known whether each of these behavior patterns is independently associated with parental stress. An examination of these differences is important in order to shed light to unique contributions of inattention, hyperactivity, and oppositional defiance on parental stress, and indicate whether specific stress and coping mechanisms are needed within the family system.

In one of the few studies of its kind, Podolski and Nigg (2001) reported that child inattention and oppositional behavior problems contributed uniquely to mother role distress. No association, however, was found between hyperactivity and mother's distress. Furthermore, research findings have focused on global parental stress reaction (Donenberg \& Baker, 1993) without shedding light on the specific contexts within the family system that are impacted

\footnotetext{
'University of Puerto Rico

${ }^{2}$ Complutense University, Spain

${ }^{3}$ Carlos Albizu University, Puerto Rico

${ }^{4}$ Clinical Neuropsychology Foundation, Argentina

${ }^{5}$ University of Michigan
}

Corresponding Author:

José J. Bauermeister, PhD, Calle Las Caobas \# 177, San Juan, Puerto Rico Email: jjbauer@prtc.net 
by inattention, hyperactivity, and ODD symptoms. For example, stressors on the family system may include difficult interactions during social activities (e.g., meetings with friends), with the school (e.g., teacher's misperceptions about parental discipline), or between family members (e.g., sibling interactions). This gap in the scientific literature is, in part, a reflection on the absence of parent-completed reports assessing the impact of their child's behavior on their families during different activities. Moreover, most of our knowledge on parental stress comes from research with Anglo-Saxon families. Cultural background can significantly influence the meaning given to ADHD and ODD type behaviors and the level of tolerance toward them. Untangling the specific effects of child behavior problems on parents is vital to improve parent-child interactions when they seek clinical care, yet must be sensitive to cultural variations in the expression of these behavior patterns (Canino \& Alegría, 2008). Consequently, as a contribution to this literature, we examine parents' reports of the impact of their children's behaviors on the family system in a community sample of Spaniards.

\section{Gender Disparities in ADHD and ODD Behaviors: Implications for Parental Stress and Coping}

The relationship between children's behavior patterns and stressors in the family system are complicated further by gender disparities in the recognition of these behaviors as maladaptive as well as the gender-specific responses to the manifestation of symptoms. Overall, recent epidemiological studies find prevalence estimates of 7\% for ADHD and 5\% for ODD (Bauermeister et al., 2007b; Bird et al., 2006; Canino et al., 2004). When stratified by gender, however, boys are found to present higher rates than girls across disorders, particularly for ADHD (Bauermeister et al., 2007a; Bird et al., 2006; Canino et al., 2004).

The role of child gender in the association between having ADHD or ODD behaviors and parental stress has received limited attention. Among the studies that have explored these studies, inconsistent findings are observed. Breen and Barkley (1988), for example, found no child gender differences in global parental stress in a clinic referred sample of children with ADHD. Bussing et al. (2003), however, reported that male gender, inattention symptoms, and having a diagnosis of ODD significantly contributed to caregiver negative impact in a school-district epidemiological sample. Podolski and Nigg (2001), on the other hand, found that parents from a sample recruited through clinics and the general community were more globally distressed if a girl presented externalizing behavior problems. These inconsistent findings, however, may be attributable to differing parental stress measures and sample characteristics across studies, and to treatment referral biases (Bauermeister et al., 2007a; Graetz, Sawyer, \& Baghurst, 2005).

Assessing the impact boys' and girls' behavior problems have on family experiences is crucial to understand which behavior patterns, and for what gender, are uniquely related to child-related parenting stress. It is also necessary for planning and implementing clinically sensitive, genderspecific family interventions and the evaluation of treatment outcomes. In response to this need, Bauermeister, Matos, and Reina (1999) developed the Family Experiences Inventory (FEI). This rating scale, similar to the one developed by Donenberg and Baker (1993) for preschool-aged children, was designed to assess caregivers' perception of the impact a child's behavior or developmental problems have on the family system. Unlike the Donenberg and Baker (1993) scale, the FEI was constructed for Puerto Rican caregivers and can assess impact of school related experiences. Factor analyses identified five factors that guided the construction of five FEI scales. These are impact on parent feelings and cognitions pertaining to: (a) relationship with the child, (b) social life of the family, (c) school, (d) financial burden, and (e) child-sibling relationships. The internal consistency of the FEI and the five scales range from .76 to .92; the test-retest reliability over a 4 to 6 week period ranged from .63 to .92 (Cumba-Avilés, Bauermeister, Martínez, Matos, \& Reina, 2008). Nonetheless, the adequacy of this psychometric scale and its underlying constructs has not been evaluated in other Spanish-speaking communities, and underscores the importance of not assuming factorial invariance across cultures or groups within a culture (Canino \& Alegría, 2008). As contribution to this literature, we examined the psychometric properties of the FEI instrument in a nonclinical sample of Spaniard families with children ages 6 to 12 years.

\section{Study Aims and Hypotheses}

This study had three overarching goals. First, we explored the factor structure and reliability of the FEI among Spaniard families. Given the longstanding influence that Spain exerted on Puerto Rico's culture and traditions, we hypothesized that the FEI factorial structure obtained from caregivers from Spain would resemble the previous Puerto Rican findings given the shared family values between the two cultures.

Second, we analyzed the perceived impact of inattention, hyperactivity, and oppositional defiant behaviors of Spaniard children on their family system, as measured by the FEI and its scales. Given our desire to assess the unique contribution of each of these behaviors on parental stress, we hypothesized that ADHD and ODD behaviors would 
have independent associations with global parent-child interactive stress. However, we anticipated that the magnitude of the relationships would vary once we examined specific contexts within the family system and accounted for the child's gender. Given the absence of literature exploring these differences and the exploratory nature of this aim, we did not have specific hypotheses across family contexts. Furthermore, based on the findings of Anastopoulos and colleagues. (1992) and Podolski and Nigg (2001), we expected that ODD behaviors would impact different aspects of family life beyond those contributed by inattention and hyperactivity symptoms.

Finally, we tested for gender differences in the association of inattention, hyperactivity, and oppositional defiant behaviors in order to assess whether family stressors were differentially related to child's gender. Given that boys may present higher rates of annoyance or distress to teachers and problems with schoolwork (Bauermeister et al., 2007a; Graetz et al., 2005) and tend to be referred to treatment more often than girls (Cabiya et al., 2006), we hypothesized a stronger male impact of inattention, hyperactivity, and oppositional defiance on parents' stress.

\section{Method}

\section{Participants}

Data were collected for 298 boys and 270 girls, ages 6 to 12 years, enrolled in four elementary schools from heterogeneously varied geographic zones in Madrid, Spain. Each school represented a different socioeconomic background as defined from indices of family income and education. Children in the sample attended schools of low (29\%), middle (39\%), and high (32\%) socioeconomic status. Within each school, one classroom per grade level was selected. If more than one student per family could participate, only one child was included in the study.

\section{Instruments}

Disruptive Behavior Rating Scale (DBRS; Barkley, Murphy, \& Bauermeister, 1998). This parent report scale includes four subscales: ADHD, Impairment, ODD, and Conduct Disorder (CD). The impairment scale is not analyzed in the present study. The ADHD subscale obtains ratings of the nine $D S M-I V$ inattention and nine hyperactivity-impulsivity symptoms, respectively. The ODD subscale obtains ratings of the eight $D S M-I V$ symptoms that define this disorder. The items in the Inattention, Hyperactivity, and ODD subscales are rated on the following 4-point scale: never or rarely (0), sometimes (1), often (2), and very often (3), using the past 6 months as a time frame. Finally, the CD subscale includes the $15 D S M-I V$ symptoms associated with this disorder. Each item is rated as present or absent during the past 12 months.

Previous findings indicated that the alpha coefficients for the Spanish version of the Inattention, Hyperactivity, and ODD subscales completed by mothers and teachers range from .86 to .96 ; the test-retest reliability indices over a 4-week period range from .78 to .89 (Bauermeister et al., 2005). For the present sample, the alpha coefficients for the Inattention, Hyperactivity, ODD, and CD subscales were $.80, .86, .80$, and .71 , respectively.

Family Experiences Inventory (FEl; Bauermeister et al., 1999). The FEI was developed to assess the positive or negative impact caretakers of children with behavior or developmental problems report to clinicians. The 51 FEI items are grouped in clinically important areas (parent feelings, social life, sibling relationships, interactions with school, finances, and relationship with partner). Parents are asked to rate the possible impact of the child's behavior or demeanor toward them and their family using a 4-point scale from Never or Almost Never (0) to Very Frequently (4) (Barkley et al., 1998). Items pertaining to sibling relationships are answered only if the child has one or more siblings aged 5 or older since these items are not developmentally appropriate for younger children. Only caretakers who are married or cohabitating answer the eight couple relationship items.

\section{Procedures}

We obtained informed consent from directors of the participating schools alongside their respective Student's Parent Associations, who authorized the research project and the administration of the assessment to parents. With the aid of school personnel, researchers sent parents of the students a letter describing the study and an invitation to answer the enclosed instruments. Parents were informed that they could do so anonymously, could return them unanswered, and could contact one of the authors (AP) to clarify concerns. Returned questionnaires were collected in a closed envelope through the child's teacher. The return rate was $87 \%$, with mothers answering $90 \%$ of the scales.

\section{Analytic Strategy}

We used a multiple imputation method for missing DBRS items (Schafer, 1999), except for those in the CD scale, given that the response format (Yes/No) of the CD items and their extremely low prevalence precluded their inclusion in the imputation process. We then performed an exploratory factor analysis of the FEI using an Alpha Factoring Extraction with a Promax rotation (Reise, Waller, \& Comrey, 2000). For the factor analysis, we used data for the 369 children with siblings 5 years or older. To mirror the 
analyses performed with the sample from Puerto Rico (Cumba-Avilés et al., 2008), we extracted five factors (excluding from the analysis the 8 items designed to assess the impact of the child's behavior on caretaker-couple relationship). We developed the five scales by selecting items with factor loadings of .30 or higher only on a given factor, and used the Cronbach's alpha coefficient to calculate reliability for each factor scale.

We examined the contribution of inattention, hyperactivity, and ODD behaviors on parent perceived impact using Multiple Linear Regression. This allowed us to determine which behavior pattern accounted for differences on FEI measures after controlling for all the other covariates in the model. Finally, we created three gender interactions for inattention, hyperactivity, and ODD behaviors, respectively, to examine the differential contribution of gender on the different aspects of family life.

\section{Results \\ Factor Analysis}

The 5-factor structure was similar to the one obtained for the FEI administered to Puerto Rican parents. These factors accounted for $43.82 \%$ of the total between-item variance. The factors were Impact on Social Life (21.76\%), Financial Burden (7.76\%), Positive Impact on Parent FeelingsCognitions (5.42\%), Impact on School Relations (4.80\%), and Impact on Siblings Feelings-Cognitions (4.09\%). Table 1 presents the items with rotated factor loadings of .30 or more for the 5 -factor solution.

\section{Development of FEI scales}

To construct the factor scales, we selected items with loadings of .30 or more only on a given factor. The first factor, Impact on Social Life, clustered feelings of disappointment with the child and cognitions about the negative impact the child's behavior has on social activities with family and friends. The second, Financial Burden, clustered cognitions about costs associated with obtaining services for the child. The third factor, positive impact on parents, clustered caretaker's feelings and cognitions indicative of satisfaction with the child's behavior, interpersonal connectedness, and personal growth. The fourth, Impact on School Relations, clustered negative cognitions about teacher's perceptions of caretaker's responsibilities managing the child's behavior and schoolwork, and school visits because of misbehavior or poor performance. The last factor, impact on siblings, clustered feelings and cognitions about child-sibling relationships, such as rejection, jealousy, and shame.

All but seven items had factor loadings greater than .40 in their respective factor (Table 1), with moderate to high
Table I. Items and Rotated (Promax) Factor Loadings for the Family Experiences Inventory

\begin{tabular}{|c|c|c|c|c|c|}
\hline \multirow[b]{2}{*}{ Items } & \multicolumn{5}{|c|}{ Factors } \\
\hline & I & 2 & 3 & 4 & 5 \\
\hline I. Does things make me feel good & & & $.59 *$ & & \\
\hline 2. Satisfied behavior management & & & $.59 *$ & & \\
\hline 3. Makes me feel ashamed & $.58 *$ & & & & \\
\hline 4. Failed as mother & .35 & & & & \\
\hline 5. Helped me be a better person & & & $.56 *$ & & \\
\hline 6. Does things to bother me & .31 & & & & \\
\hline 7. Behavior not expected for a son & $.5 I^{*}$ & & & & \\
\hline 8. Live pleasant moments with him & & & $.56 *$ & & \\
\hline 9. Understand each other & & & $.57 *$ & & \\
\hline 10.Angry way he is & .56 & & & & \\
\hline II.Thankful for things I do & & & $.66 *$ & & \\
\hline 12. Sad for the way he is & .64 & & & & \\
\hline I3. I can't enjoy recreation & $.4 I^{*}$ & & & & \\
\hline I4. I can't carry out responsibilities & $.48 *$ & & & & \\
\hline I5. I can't spend time with family & $.65^{*}$ & & & & \\
\hline I6. I can't spend time with friends & $.73 *$ & & & & \\
\hline 17. Others don't approve his behavior & $.57 *$ & & & & \\
\hline 18. Family doesn't participate activities & $.54 *$ & & & & \\
\hline \multicolumn{6}{|l|}{ 19. Difficulty getting babysitter } \\
\hline 20. Difficult going shopping & $.59 *$ & & & & \\
\hline 21. Teachers think not a good mother & & & & $.35 *$ & \\
\hline 22.Visit school due to behavior & & & & .44 & \\
\hline 23. Visit school due to schoolwork & & & & .41 & \\
\hline \multicolumn{6}{|l|}{ 24. Sit with him for homework } \\
\hline \multicolumn{6}{|l|}{ 25. Tension when homework } \\
\hline 26. Rejected by classmates & & & & .33 & \\
\hline 27. School provides what he needs & & & .51 & & \\
\hline 28. Teachers understand him & -.35 & & .58 & .41 & \\
\hline 29. Don't teach him to behave & & & & $.52 *$ & \\
\hline 30. Don't attend to his schoolwork & & & & $.45 *$ & \\
\hline 31. More medical services & & $.72 *$ & & & \\
\hline 32. More medication & & $.72 *$ & & & \\
\hline 33. More mental health services & & $.77 *$ & & & \\
\hline 34. More educational help & & $.59 *$ & & & \\
\hline 35. Absent from work/chores & & $.65^{*}$ & & & \\
\hline 36. Pay for services & & $.59 *$ & & & \\
\hline 37. Siblings get along with him & & & & & $.31 *$ \\
\hline 38. Siblings complain about him & & & & & $.39 *$ \\
\hline 39. Siblings ashamed of him & & & & & $.53 *$ \\
\hline 40. Siblings reject him & & & & & $.64 *$ \\
\hline 4I. Siblings annoyed by time with him & & & & & $.46 *$ \\
\hline 42. Siblings think he is the favorite one & & & & & $.61 *$ \\
\hline 43. Siblings tell him not to bother me & & & & & $.37 *$ \\
\hline
\end{tabular}

Note: Factor loadings less than .30 are not included. Factor I = Impact on Social Life; Factor 2 = Financial Burden; Factor 3 = Positive Impact on Parent Feelings-Cognitions; Factor $4=$ Impact on School Relations; Factor $5=$ Impact on Siblings Feelings-Cognitions. Items with factor loadings in bold were selected for the factor scales; items with an asterisk loaded on the same factors as in the Puerto Rican sample.

Cronbach's alpha coefficients for the Total (39 items; $\alpha=$ .89), Impact on Social Life (13 items; $\alpha=.85$ ), Financial Burden ( 6 items; $\alpha=.82$ ), Positive Impact on Parent 
Table 2. Means and Standard Deviations for Study Measures by Gender

\begin{tabular}{|c|c|c|c|c|}
\hline & Females & Males & Total & $t$ or $\chi^{2}$ statistic \\
\hline \multicolumn{5}{|l|}{ Child characteristics } \\
\hline Age (in years) & $8.73(1.88)$ & $8.68(1.83)$ & $8.70(1.85)$ & 0.33 \\
\hline Hyperactivity & $5.4 I(4.48)$ & $6.59(5.21)$ & $6.03(4.91)$ & $-2.91 *$ \\
\hline Inattention & $6.03(4.21)$ & $6.69(4.20)$ & $6.38(4.22)$ & -1.89 \\
\hline Oppositional defiance & $4.16(3.42)$ & $4.65(3.64)$ & $4.42(3.54)$ & -1.67 \\
\hline \multicolumn{5}{|l|}{ FEI scales } \\
\hline Total FEI & $12.19(9.59)$ & $12.18(9.99)$ & $12.18(9.79)$ & 0.01 \\
\hline Impact on Social Life & $2.20(3.75)$ & $2.53(3.5 I)$ & $2.37(3.63)$ & -1.06 \\
\hline Financial Burden & $1.00(2.20)$ & $1.24(2.54)$ & $1.13(2.38)$ & -1.17 \\
\hline Positive impact on parents & $15.79(3.75)$ & $16.03(3.4 I)$ & $15.91(3.57)$ & -0.80 \\
\hline School relations & $0.84(I .4 I)$ & $1.03(1.82)$ & $0.94(1.64)$ & -1.42 \\
\hline Impact on siblings & $2.97(2.50)$ & $2.78(2.60)$ & $2.87(2.55)$ & 0.70 \\
\hline Couples relationship & $2.23(2.68)$ & $2.67(3.11)$ & $2.47(2.92)$ & -1.39 \\
\hline
\end{tabular}

Note: Standard deviations in parenthesis. FEI = Family Experiences Inventory

$* p<.05$

(7 items; $\alpha=79$ ), Impact on School Relations (6 items; $\alpha=$ .63 ), and Impact on Siblings ( 7 items; $\alpha=.70$ ) scales. We also constructed an Impact on Couple Relationship scale ( 8 items; $\alpha=.75$ ). As an exploratory step, we computed the reliability for the FEI scales separately for males and females to ensure that gender did not confound the internal consistency of the subscales. With the exception of the Impact on School Relations scale, where the coefficient was higher for males (.72) and lower for girls (.48), we found the reliability coefficient for all FEI subscales was similar when stratified by gender.

\section{Child Characteristics and FEI scores}

As shown in Table 2, boys and girls did not differ on age, parental ratings of inattention and oppositional defiant behaviors, or on the FEI and its scales' scores. Boys, however, were rated as more hyperactive than girls $(p<.05)$.

\section{Contribution of ADHD and ODD Symptoms to FEl scores}

We used multiple linear regression analyses to examine the contribution of inattention, hyperactivity and oppositional defiance on family impact. In these regressions, we explored the main effects of SES, gender, and Hyperactivity, Inattention, and ODD scale scores, after accounting for the differential contribution of gender on the Hyperactivity, Inattention, and ODD scores. Given the relatively weak bivariate association of CD scale scores with the FEI scale scores (i.e., Impact on Social Life $[r=.15 ; p<.01]$, Positive Impact on Parents $[r=-.09 ; p<.05]$, and Impact on Siblings $[r=.11 ; p<.05]$ scales), respectively, we did not enter $\mathrm{CD}$ scores in the regression analyses. Below, we present a summary of the analyses by FEI outcome.
Total FEl. As shown in Table 3, our model accounted for $11 \%$ of the total variance in Total FEI score $[F(8,360)=$ $6.65, p<.001]$. The Total FEI score is the sum of all factor scales scores and included families with participants who had one or more siblings aged 5 or older $(N=369)$. We found that oppositional defiant behaviors were positively associated with Total FEI, after controlling for all other covariates in the model. While the main effects for gender and hyperactivity were nonsignificant, we found gender had a differential effect on the association between Hyperactivity and Total FEI score. Inspection of the data showed that girls with higher Hyperactivity scale scores were more likely to score lower on the FEI Total score. Boys with higher Hyperactivity scores, on the other hand, were more likely to score higher on the FEI Total score. No other statistically significant main or interaction effects were found.

Impact on Social Life. We accounted for $17 \%$ of the total variance in our measure of Impact on Social Life $[F(8,548)$ $=14.94, p<.001]$. A higher ODD scale score was associated with a higher Impact on Social Life score, after controlling for all other covariates in the model. We found no other statistically significant main or interaction effects.

Financial Burden. The full model accounted for $5 \%$ of the total variance in our measure of Financial Burden $[F(8$, $544)=4.68, p<.01]$. A higher ODD score was associated with a higher score for Financial Burden, after controlling for all other covariates in the model. We found no other statistically significant main or interaction effects.

Positive impact on parents. Our model accounted for $12 \%$ of the total variance in our measure of Positive Impact on Parents $[F(8,542)=9.48, p<.001]$. We found higher SES was positively associated with higher scores in the Positive Impact on Parents subscale. Conversely, we found that higher scores in Inattention and ODD scale scores, respectively, were negatively associated with Positive 
Table 3. Regression Analyses for Demographic and Child Variables Predicting Family Impact

\begin{tabular}{|c|c|c|c|}
\hline Variable & $B$ & SEB & $\beta$ \\
\hline \multicolumn{4}{|l|}{ Total FEl $R^{2}=.1 \mathrm{I}$} \\
\hline SES & -1.34 & 0.99 & -0.07 \\
\hline Gender & -1.62 & 1.90 & -0.08 \\
\hline Hyperactivity & -0.33 & 0.22 & -0.15 \\
\hline Inattention & 0.43 & 0.24 & 0.18 \\
\hline Oppositional Defiant & 0.79 & 0.23 & $0.29 * * *$ \\
\hline Hyperactivity $\times$ Gender & 0.97 & 0.30 & $0.43 * * *$ \\
\hline Inattention $\times$ Gender & -0.52 & 0.32 & -0.23 \\
\hline Oppositional Defiant $\times$ Gender & -0.27 & 0.29 & -0.10 \\
\hline \multicolumn{4}{|l|}{ Social Life $R^{2}=.17$} \\
\hline SES & -0.50 & 0.29 & -0.07 \\
\hline Gender & 0.07 & 0.57 & 0.01 \\
\hline Hyperactivity & -0.02 & 0.06 & -0.03 \\
\hline Inattention & 0.13 & 0.07 & 0.15 \\
\hline Oppositional Defiant & 0.38 & 0.07 & $0.37 * * *$ \\
\hline Hyperactivity $\times$ Gender & 0.14 & 0.08 & 0.19 \\
\hline Inattention $\times$ Gender & -0.07 & 0.09 & -0.08 \\
\hline Oppositional Defiant $\times$ Gender & -0.10 & 0.09 & -0.10 \\
\hline \multicolumn{4}{|l|}{ Financial Burden $R^{2}=.05$} \\
\hline SES & 0.21 & 0.20 & 0.04 \\
\hline Gender & -0.46 & 0.40 & -0.10 \\
\hline Hyperactivity & -0.07 & 0.05 & -0.14 \\
\hline Inattention & 0.08 & 0.05 & 0.14 \\
\hline Oppositional Defiant & 0.11 & 0.05 & $0.16 *$ \\
\hline Hyperactivity $\times$ Gender & 0.10 & 0.06 & 0.20 \\
\hline Inattention $\times$ Gender & 0.001 & 0.07 & 0.001 \\
\hline Oppositional Defiant $\times$ Gender & 0.001 & 0.06 & 0.01 \\
\hline \multicolumn{4}{|l|}{ Positive Impact on Parents $R^{2}=.12$} \\
\hline SES & 0.68 & 0.29 & $0.10 *$ \\
\hline Gender & 0.52 & 0.58 & 0.07 \\
\hline Hyperactivity & 0.10 & 0.07 & 0.13 \\
\hline Inattention & -0.15 & 0.07 & $-0.17^{*}$ \\
\hline Oppositional Defiant & -0.29 & 0.09 & $-0.29 * *$ \\
\hline Hyperactivity $\times$ Gender & -0.23 & 0.09 & $-0.32 * *$ \\
\hline Inattention $\times$ Gender & 0.18 & 0.10 & 0.22 \\
\hline Oppositional Defiant $\times$ Gender & 0.05 & 0.09 & 0.05 \\
\hline \multicolumn{4}{|l|}{ School Relations $R^{2}=.06$} \\
\hline SES & -0.22 & 0.14 & -0.07 \\
\hline Gender & -0.09 & 0.27 & -0.03 \\
\hline Hyperactivity & -0.07 & 0.03 & -0.22 \\
\hline Inattention & 0.09 & 0.03 & $0.23 * *$ \\
\hline Oppositional Defiant & 0.07 & 0.03 & $0.15^{*}$ \\
\hline Hyperactivity $\times$ Gender & 0.14 & 0.04 & $0.43 * * *$ \\
\hline Inattention $\times$ Gender & -0.07 & 0.05 & -0.18 \\
\hline Oppositional Defiant $\times$ Gender & 0.04 & 0.04 & -0.09 \\
\hline \multicolumn{4}{|l|}{ Siblings $R^{2}=.03$} \\
\hline SES & 0.07 & 0.27 & 0.01 \\
\hline Gender & -0.36 & 0.52 & -0.07 \\
\hline Hyperactivity & -0.08 & 0.06 & -0.13 \\
\hline Inattention & 0.05 & 0.07 & 0.09 \\
\hline Oppositional Defiant & 0.16 & 0.06 & $0.22 * *$ \\
\hline Hyperactivity $\times$ Gender & 0.19 & 0.08 & $0.33 *$ \\
\hline Inattention $\times$ Gender & -0.11 & 0.09 & -0.19 \\
\hline Oppositional Defiant $\times$ Gender & -0.08 & 0.08 & -0.11 \\
\hline
\end{tabular}

Table 3. (continued)

\begin{tabular}{lrrr}
\hline Variable & $B$ & SEB & $\beta$ \\
\hline Couple Relationships $R^{2}=. I I$ & & & \\
$\quad$ SES & 0.06 & $0.3 \mathrm{I}$ & 0.01 \\
Gender & 0.33 & 0.60 & 0.06 \\
Hyperactivity & 0.06 & 0.07 & 0.10 \\
Inattention & -0.03 & 0.08 & -0.04 \\
Oppositional Defiant & 0.25 & 0.08 & 0.30 *** \\
Hyperactivity $\times$ Gender & 0.09 & 0.10 & 0.14 \\
Inattention $\times$ Gender & -0.10 & 0.10 & -0.15 \\
Oppositional Defiant $\times$ Gender & -0.01 & 0.09 & -0.10 \\
\hline
\end{tabular}

Note: $\mathrm{FEI}=$ Family Experiences Inventory; $R^{2}=$ adjusted $R$-square; $\mathrm{SES}=$ socioeconomic status.

$*_{p}<.05$. **p $<.01$. *** $p<.001$.

Impact on Parents, after controlling for all other covariates in the model.

While the main effects for gender and hyperactivity were nonsignificant, we found gender had a differential effect on the association between Hyperactivity scores and Positive Impact on Parents. Girls with higher Hyperactivity scores were more likely to score higher on the Positive Impact on Parents score. Boys with higher Hyperactivity scores, on the other hand, were less likely to score higher on the Positive Impact on Parents score. No other statistically significant main or interaction effects were found.

School relations. We accounted for $6 \%$ of the total variance in our measure of School Relations $[F(8,543)=5.52$, $p<.001]$. We found higher scores in Inattention and ODD scales, respectively, were positively associated with School Relations, after controlling for all other covariates in the model. While the main effects for gender and Hyperactivity were nonsignificant, we found gender had a differential effect on the association between Hyperactivity and School Relations. Following a similar trend to that reported for Total FEI scores, girls with higher Hyperactivity scores were more likely to score lower on the School Relations score. Boys with higher Hyperactivity scores, on the other hand, were more likely to score higher on the School Relations score. No other statistically significant main or interaction effects were found.

Impact on siblings. For this analysis, we selected only those families with participants' who had one or more siblings aged 5 or older $(N=369)$, accounting for only $3 \%$ of the total variance in the Impact on Siblings score $[F(8,360)=2.44, p<$ $.05]$. We found ODD scale scores were positively associated with Impact on Siblings, after controlling for all other covariates in the model. While the main effects for gender and hyperactivity were nonsignificant, we found gender had a differential effect on the association between Hyperactivity scores and Impact on Siblings. Following a similar trend, girls with higher Hyperactivity scores were more likely to score lower on the Siblings scale. Boys with higher Hyperactivity 
scores, on the other hand, were more likely to score higher on the scale. We found no other statistically significant main or interaction effects on the Impact on Siblings scale.

Impact on couple's relationship. For this analysis, we selected only those parents who reported being in a relationship $(N=343)$. The full model accounted for $11 \%$ of the total variance in our measure of Impact on Couple's Relationship $[F(8,342)=6.26, p<.001]$. A higher ODD scale score was associated with a higher impact on a couple's relationship, after controlling for all other covariates in the model. We found no other statistically significant main or interaction effects.

\section{Discussion}

The first goal of our study was to examine the factorial structure of the FEI in a community sample of Spaniard parents (mostly mothers). Consistent with our first hypothesis, we found that the factor structure obtained in this sample is similar to that obtained for Puerto Rican mothers (Cumba-Avilés et al., 2008). Similarly to the scales developed for children in Puerto Rico, the factorially derived scales and the 8-item scale on couple relationships have moderate to high internal consistency. The Impact on School Relations scale, however, yielded a higher reliability for boys than for girls. The diminished reliability across genders in the School Relation scale suggests a need to readdress what constitutes a "problem" for boys and girls, respectively, and whether the manifestation of these symptoms at school may be less permissive for boys than girls. The reasons behind this finding need to be identified in further research.

As to the second goal of our study, our findings are consistent with previous research that documents the increased caretaking demands and global impact on family life associated with raising a child with inattention, hyperactivity, and oppositional defiance (Anastopoulos et al., 1992; Harrison \& Sofronoff, 2002; Podolski \& Nigg, 2001). This finding has clinical significance because it relates to children from a nonreferred sample of Spaniard families and expands our scientific knowledge about ADHD and ODD behaviors to a community sample from a different national group. Consistent with our second hypothesis, ODD symptoms were the only set of behaviors that were uniquely and positively correlated with impact in global child-related parenting stress, over and above the impact of inattention and hyperactivity. ODD symptoms are related to presence of negative and hostile feelings, and defiant, argumentative, and disobedient behaviors likely to have a strong impact on parents and authority figures. Our findings support previous reports (Anastopoulos et al., 1992; Bussing et al., 2003; Podolski \& Nigg, 2001) and reiterate the potent role conduct problem behaviors play, in addition to ADHD symptoms, on quality of family life in general and on family stress in particular (Klassen, Miller, \& Fine, 2004).
As expected from our second hypothesis, the pattern of unique associations for inattention, hyperactivity and oppositional defiance varied depending upon the behavior and dimension of family life examined. These findings have not been reported previously probably because researchers have concentrated on studying global measures of parental stress and have not asked parents directly about the impact of their child's behavior on their families across different dimensions (Donenberg \& Baker, 1993). Severity of oppositional defiance was uniquely and positively correlated with impact in all the dimensions evaluated in the FEI. Hyperactivity had a differential gender impact on global child-related parenting stress. Further analyses indicated that this unique association was obtained only on parents' perception of the impact of severity of boys' hyperactivity in school and sibling relations, respectively, and in their positive feelings and cognitions toward the child's behavior (see below). Finally, severity of inattention had a unique association with perceived higher negative impact on school related activities and lower positive impact on parents. These inattention findings are consistent with reports that attention processes are associated with learning and academic performance (Barkley, 2006; Lahey et al., 1994, 1998) and the importance parents bestow on their children's school success. In brief, our findings support the hypothesis that inattention, hyperactivity, and oppositional defiance may have unique and differential impact on family life and contribute to childrelated parent stress in some contexts but not others, and point out to the complexity of parent-child interactive stress.

The role of child gender in the relationship between ADHD/ODD behaviors and parental stress has received limited attention in the research literature and has yielded inconsistent results. Our findings indicate that, compared to girls, boys with higher hyperactivity scores were more likely to score higher on the FEI Total, School Relations, and Siblings score, and more likely to score lower on the Positive Impact on Parents score. These findings suggest that parents perceive greater child-related stress and place greater burden from having a male child with hyperactivity. Interestingly, we found no male gender-hyperactivity interactions in areas such as social life, financial burden, and couple relationship.

Also contrary to our expectations, we did not find genderoppositional defiance or gender-inattention interactions. For these behavior patterns, boys with higher scores were not more likely than girls to score higher on the FEI. Further research is needed to understand why only a genderhyperactivity interaction is found in some areas of family function but not others. Research in this area is important, because it can shed light on why more boys than girls with ADHD are treated or suspended/expelled from school (Bauermeister et al., 2007a).

Our study faces several methodological shortcomings. First, we did not have independent measures to confirm 
mothers' reports of the child's ADHD and ODD behaviors (e.g., direct observations, teachers' reports) or family impact (e.g., financial burden). In addition, fathers' reports may have provided different perceptions of their children's behaviors and impact on family life (Barkley, 2006; Christensen, Margolin, \& Sullaway, 1992). Second, we only included children's behaviors and sociodemographic characteristics in the estimation of stressors within the family system. Consequently, an inspection of our regression models may suggest that our model did not explain a sufficient portion of the variance for each FEI scale estimated (i.e., $R^{2}$ ranged between $3 \%$ and $17 \%$ ). Nonetheless, rather than signifying that our model was an inadequate fit, the limited explained variance underscores the complexity of family dynamics and suggests that additional covariates (e.g., parent-child interactions) be considered in future research. Finally, given the cross-sectional design of our study, it is not possible to determine a causal relationship between the child's behavior and perceived family impact. Although the research literature on the etiology of ADHD and ODD, as well as clinical experience, support the interpretation that symptoms of these disorders have a stressful impact, longitudinal research using the FEI or similar measures is needed to confirm this conceptualization.

\section{Conclusions}

Taken together, our findings highlight the need for clinicians to understand that, although ADHD and ODD symptoms have a global impact on the family, these behaviors are correlated with impact on some aspects of family experiences but not others. Clinical and research studies need to assess specific areas of family impact and not only global parent-child related stress. Understanding of these issues is also critical for planning and implementing prevention, parent support, and clinically sensitive family treatment programs and evaluating intervention outcomes.

\section{Acknowledgments}

We acknowledge the collaboration of the directors and faculty of Alarcón, Estudio, Guindalera, and Lucero schools, alongside their respective Student's Parent Associations, as well as Colegio Inmaculada Concepción. We also wish to thank participating parents for completing the questionnaires and the assistance of Grace Reina and Mariano Scándar.

\section{References}

American Psychiatric Association. (1994). Diagnostic and statistical manual of mental disorders (4th ed.). Washington, DC: Author.

Anastopoulos, A. D., Guevremont, D. C., Shelton, T. L., \& DuPaul, G. J. (1992). Parenting stress among families of children with attention deficit hyperactivity disorder. Journal of Abnormal Child Psychology, 20, 503-520.
Barkley, R. A. (2006). Attention-deficit hyperactivity disorder. A handbook for diagnosis and treatment (3rd ed.). New York: Guilford.

Barkley, R. A., Murphy, K. R., \& Bauermeister, J. J. (1998). Trastorno por Déficit de Atención e Hiperactividad. New York: Guilford.

Bauermeister, J. J. (1992). Factor analyses of teacher ratings of attention deficit-hyperactivity and oppositional defiant symptoms in children aged four through thirteen years. Journal of Clinical Child Psychology, 21, 27-34.

Bauermeister, J. J., Matos, M., \& Reina, G. (1999). Do the combined and inattentive types of ADHD have a similar impact on family life? ADHD Report, 7, 6-8.

Bauermeister, J. J., Matos, M., Reina, G., Salas, C. C., Martínez, J. V., Cumba, E., et al. (2005). Comparison of the DSM-IV combined and inattentive types of ADHD in a school-based sample of Latino/Hispanic children. Journal of Child Psychology and Psychiatry, 46, 166-179.

Bauermeister, J. J., Shrout, P. E., Chávez, L., Rubio-Stipec, M., Ramírez, R., Padilla, L., et al. (2007a). ADHD and gender: Are risks and sequela of ADHD the same for boys and girls? Journal of Child Psychology and Psychiatry, 48, 831-839.

Bauermeister, J. J., Shrout, P. E., Ramírez, R., Bravo, M., Alegría, M., Martínez-Taboas, A., et al. (2007b). ADHD correlates, comorbidity and impairment in community and treated samples of children and adolescents. Journal of Abnormal Child Psychology, 35, 883-898.

Bird H. R., Davies M., Duarte, C. S., Shen S., Loeber, R., \& Canino, G.(2006). A study of disruptive behavior disorders in Puerto Rican youth: II. Baseline prevalence, comorbidity, and correlates in two sites. Journal of the American Academy of Child and Adolescent Psychiatry, 45, 1042-1053.

Breen, M. J., \& Barkley, R. A. (1988). Child psychopathology and parenting stress in girls and boys having attention deficit disorder with hyperactivity. Journal of Pediatric Psychology, 13, 265-280.

Bussing, R., Gary, F., Mason, D. M., Leon, C. E., Sinha, K., \& Garvan, C. W. (2003). Child temperament, ADHD, and caregiver strain: exploring relationships in an epidemiological sample. Journal of the American Academy of Child and Adolescent Psychiatry, 42, 184-192.

Cabiya, J. J., Canino, G., Chávez, L., Ramírez, R., Alegría, M., Shrout, P. et al. (2006). Gender disparities in mental health service use of Puerto Rican children and adolescents. Journal of Child Psychology and Psychiatry, 47, 840-848.

Canino, G., \& Alegría, M. (2008). Psychiatric diagnosis-is it universal or relative to culture? Journal of Child Psychology and Psychiatry, 49, 237-250.

Canino G., Shrout, P. E., Rubio-Stipec, M., Bird, H. R., Bravo, M., Ramírez, R., et al. (2004). The DSM-IV rates of child and adolescent disorders in Puerto Rico: prevalence, correlates, service use, and the effects of impairment. Archives of General Psychiatry, 61, 85-93. 
Christensen, A., Margolin, G., \& Sullaway, M. (1992). Interparental agreement on child behavior problems. Psychological Assessment, 4, 419-425.

Cumba-Avilés, E., Bauermeister, J. J., Martínez, J. V., Matos, M., \& Reina, G. (2008). Factor structure and psychometric properties of the Family Experiences Inventory. Manuscript submitted for publication.

Donenberg, J. G., \& Baker, B. L. (1993). The impact of young children with externalizing behaviors on their families. Journal of Abnormal Child Psychology, 21, 179-198.

Graetz, B. W., Sawyer, M. G., \& Baghurst, P. (2005). Gender differences among children with DSM-IV ADHD in Australia. Journal of the American Academy of Child and Adolescent Psychiatry, 44, 159-168.

Harrison, C., \& Sofronoff, K. (2002). ADHD and parental psychological distress: role of demographics, child behavioral characteristics, and parental cognitions. Journal of the American Academy of Child and Adolescent Psychiatry, 41, 703-711.

Hinshaw, S. P. (1987). On the distinction between attentional deficits/hyperactivity and conduct problems/aggression in child psychopathology. Psychological Bulletin, 101, 443-463.

Hinshaw, S. P., \& Lee, S. S. (2003). Conduct and oppositional defiant disorders. In E. J. Mash \& R. A. Barkley (Eds.), Child Psychopathology (2nd ed., pp. 144-198). New York: Guilford.

Klassen, A. F., Miller, A., \& Fine, S. (2004). Health-related quality of life in children and adolescents who have a diagnosis of attention-deficit/hyperactivity disorder. Pediatrics, 114, e541-e547.

Lahey, B. B., Applegate, B., McBurnett, K., Biederman, J., Greenhill, L., Hynd, G. W., et al. (1994). DSM-IV field trials for attention deficit/hyperactivity disorder in children and adolescents. Journal of the American Academy of Child and Adolescent Psychiatry, 151, 1673-1685

Lahey, B. B., Pelham, W. E., Stein, M.A., Loney, J., Trapani, C., Nugent, K., et al. (1998). Validity of DSM-IV attention-deficit/ hyperactivity disorder for younger children. Journal of the American Academy of Child and Adolescent Psychiatry, 37, 695-702.

Pelham, W. E., Gnagy, E. M., Greenslade, K. E., \& Milich, R. (1992). Teacher ratings of DSM-III-R symptoms for the disruptive behavior disorders. Journal of the American Academy of Child and Adolescent Psychiatry, 31, 210-218.
Podolski, C., \& Nigg, J. T. (2001). Parent stress and coping in relation to child ADHD severity and associated child disruptive behavior problems. Journal of Clinical Child Psychology, 30, 503-513.

Reise, S. P., Waller, N., \& Comrey, A. L. (2000). Factor analysis and scale revision. Psychological Assessment, 12, 287-297.

Schafer, J. L. (1999). Multiple imputation: A primer. Statistical Methods in Medical Research, 8, 3-15.

Wolraich, M. L., Lambert, E. W., Baumgaertel, A., Garcia-Tornel, S., Feurer, I. D., Bickman, L., et al. (2003). Teachers' screening for attention deficit/hyperactivity disorder: comparing multinational samples on teacher ratings of ADHD. Journal of Abnormal Child Psychology, 31, 445-455.

\section{Bios}

José J. Bauermeister, $\mathrm{PhD}$, is retired professor of Psychology and Researcher at the Behavioral Sciences Research Institute, University of Puerto Rico, Puerto Rico. His program of research concentrates on conceptualization and treatment of ADHD, as well as cultural factors.

Aníbal Puente, $\mathrm{PhD}$, is professor in the Faculty of Psychology and Institute of Biofunctional Studies, Complutense University, Spain. His research focuses on reading, learning disabilities, and metacognition.

José V. Martínez, PhD, is profesor of statistics and psychology in the Program in Clinical Psychology, Carlos Albizu University, Puerto Rico. His research focuses on ADHD and storytelling techniques assessment in school age children.

Eduardo Cumba, $\mathrm{PhD}$, is researcher at The Institute for Psychological Research, University of Puerto Rico, Puerto Rico. His research program focuses on ADHD and treatment of depression in adolescents.

Rubén O. Scándar, MA, is professor of psychology at the Clinical Neuropsychology Foundation, Argentina. His research focus is on ADHD, learning disabilities, and clinical neuropsychology.

José A. Bauermeister, MPH, PhD, is Assistant Research Professor, Health Behavior and Health Education, School of Public Health, University of Michigan, Ann Arbor. His research program is on prevention and health promotion strategies for high-risk adolescents and young adults. 